\title{
Hafnium films and magnetic shielding for TIME, a mm-wavelength spectrometer array
}

\author{
J. Hunacek ${ }^{a}$. J. Bock ${ }^{a, b}$. C.M. Bradford ${ }^{b, a}$. \\ V. Butler ${ }^{f}$. T.-C. Chang ${ }^{b}$. Y.-T. Cheng ${ }^{a}$. \\ A. Cooray $^{d}$. A. Crites ${ }^{a}$. C. Frez $^{b}$. \\ S. Hailey-Dunsheath ${ }^{a}$ - B. Hoscheit ${ }^{a}$. D.W. Kim ${ }^{e}$. \\ C.-T. $\mathbf{L i}^{c}$. D. Marrone ${ }^{e}$. L. Moncelsi ${ }^{a}$. \\ E. Shirokoff ${ }^{g}$. B. Steinbach ${ }^{a}$. G. Sun ${ }^{a}$. \\ I. Trumper ${ }^{e}$ A. Turner ${ }^{b}$ B. Uzgil ${ }^{h} \cdot$ A. Weber ${ }^{b}$. \\ M. Zemcov ${ }^{f}$
}

the date of receipt and acceptance should be inserted later

\begin{abstract}
TIME is a mm-wavelength grating spectrometer array that will map fluctuations of the $157.7 \mu \mathrm{m}$ emission line of singly ionized carbon ([CII]) during the Epoch of Reionization (redshift $\mathrm{z} \sim 5$ to 9). 60 transition-edge sensor (TES) bolometers populate the output arc of each of the 32 spectrometers, for a total of 1920 detectors. Each bolometer consists of gold absorber on $\mathrm{a} \sim 3 \mathrm{x} 3 \mathrm{~mm}$ silicon nitride micro-mesh suspended near the corners by $1 \mathrm{x} 1 \mathrm{x}$ $500 \mu \mathrm{m}$ silicon nitride legs targeting a photon-noise-dominated NEP $\sim 1 \times 10^{-17} \mathrm{~W} / \sqrt{\mathrm{Hz}}$. Hafnium films are explored as a lower- $T_{c}$ alternative to Ti $(500 \mathrm{mK})$ for TIME TESs, allowing thicker support legs for improved yield. Hf $T_{c}$ is shown to vary between $250 \mathrm{mK}$ and $450 \mathrm{mK}$ when varying the resident Ar pressure during deposition. Magnetic shielding designs and simulations are presented for the TIME first-stage SQUIDs. Total axial field suppression is predicted to be $5 \times 10^{7}$.
\end{abstract}

Keywords spectrometers, bolometers, transition edge sensors, hafnium, magnetic shielding

\section{Introduction}

TIME $^{1,2,3}$ is a mm-wavelength spectrometer array ${ }^{4}$ that will map fluctuations of the 157.7 $\mu \mathrm{m}$ emission line of singly ionized carbon ([CII]) during the Epoch of Reionization (redshift $\mathrm{z} \sim 5$ to 9$)^{5,6}$. A $14 \mathrm{x} 0.43$ arcmin instantaneous field of view corresponding to $16 \mathrm{x}$ 1 spatial pixels is sampled by two banks single-polarization grating spectrometers ( $32 \mathrm{spec}-$ trometers total). Each spectrometer consists of an input feedhorn, a parallel-plate waveguide, and a curved diffraction grating (similar to that used in Z-Spec ${ }^{7,8}$ ) with resolving power $\mathrm{R}$

E-mail: jhunacek@caltech.edu

${ }^{a}$ California Institute of Technology, Pasadena, USA

${ }^{b}$ Jet Propulsion Laboratory, Pasadena, USA

${ }^{c}$ Academia Sinica Institute of Astronomy and Astrophysics, Taipei, Taiwan

${ }^{d}$ University of California Irvine, Irvine, USA

${ }^{e}$ University of Arizona, Tucson, USA

${ }^{f}$ Rochester Institute of Technology, Rochester, USA

${ }^{g}$ University of Chicago, Chicago, USA

${ }^{h}$ Max Planck Institute for Astronomy, Heidelberg, Germany 
$=170$ and spectral range 183 to $326 \mathrm{GHz}$. The output arc of each spectrometer is sampled at $\mathrm{R} \sim 100$ with 60 TES bolometers, of which 16 on the band edges are used for atmospheric monitoring and removal. The TESs (1920 total) are designed in close-packed buttable arrays of 8 spatial x 12 spectral (high frequency) or 8 spatial x 8 spectral (low frequency) pixels, and will be operated from a $250 \mathrm{mK}$ base temperature with a photon-noise-dominated NEP $\sim 1 \times 10^{-17} \mathrm{~W} / \sqrt{\mathrm{Hz}}$. Each bolometer consists of gold absorber on a $2.3 \times 3 \mathrm{~mm}$ (high frequency) or $3.5 \times 3 \mathrm{~mm}$ (low frequency) silicon nitride micro-mesh suspended near the corners by $1 \times 1 \times 500 \mu \mathrm{m}$ silicon nitride legs. Absorbed radiation is thermally coupled to elemental $\mathrm{Al}$ and $\mathrm{Ti}$ TESs connected in series ( $\mathrm{Al}$ is used in higher-loading lab conditions). Detector readout uses SQUIDs and time-domain multiplexing. Prototype TIME high frequency detector arrays with fully-released micro-meshes can be seen in Fig. 1.

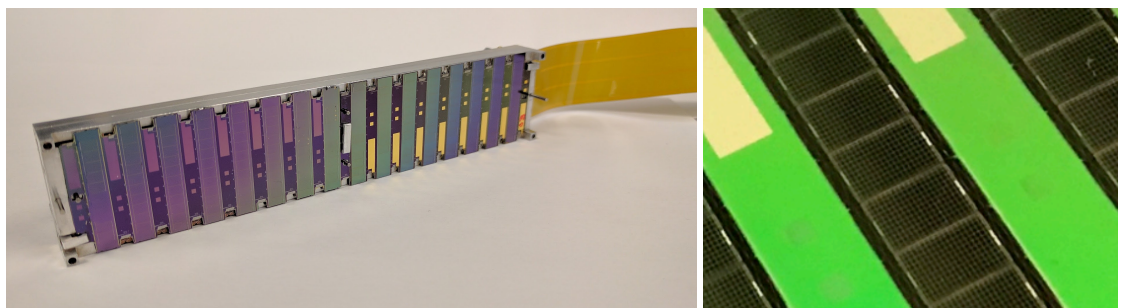

Fig. 1 Left: A prototype TIME high frequency detector module, responsible for 12 spectral channels for each of 16 spectrometers. Right: Fully-released silicon nitride micro-mesh absorbers in a prototype TIME high frequency detector sub-module.

\section{Hafnium Films}

Current TIME detectors use a Ti TES with $T_{c}=495 \mathrm{mK}$. Because we operate at a base temperature of $250 \mathrm{mK}$, reducing to $T_{c} \sim 350 \mathrm{mK}$ would allow us to use $4 \mathrm{x}$ thicker suspension legs for the micro-mesh absorbers at the same saturation power without phonon noise penalties, thus improving device yield and durability. Toward that end, $160 \mathrm{~nm} \mathrm{Hf}$ films were deposited on silicon nitride coated silicon wafers at the JPL Microdevices Laboratory (MDL). Resident argon pressure in the deposition chamber was controlled between 2.5 and 15 mTorr during the sputtering process for different samples to influence the stress of the resulting films. Results are shown in Fig. 2. Films had a room temperature resistivity of approximately $5.5 \Omega / \square$. $T_{c}$ was found to vary between 250 and $450 \mathrm{mK}$ with good centerto-edge uniformity, indicating that the desired $350 \mathrm{mK}$ is achievable with Hf. TIME-style devices with Hf TESs are being processed and will be tested in the near future.

\section{Magnetic Shielding}

\subsection{First Stage SQUID Module Enclosures}

The first-stage SQUIDs in TIME are located on 12 PCB daughtercards at the $250 \mathrm{mK}$ stage, each of which is responsible for either 192 or 128 TES bolometers (32 rows x 6 columns for high frequency modules, 32 rows x 4 columns for low frequency). Three 37 pin micro-D connectors mate to the signal distribution motherboard, and a set of superconducting flexcircuits (one per multiplexing column) carries traces to the detectors. Each daughtercard is 

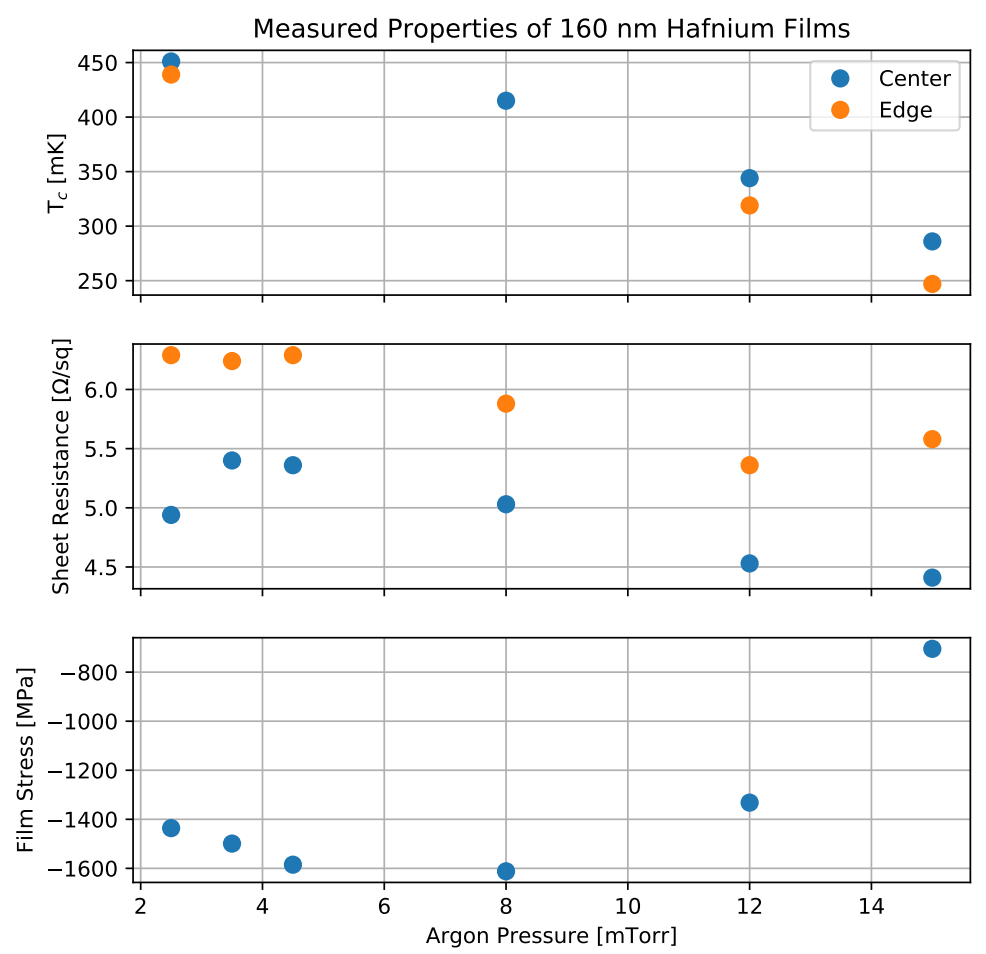

Fig. 2 Measured properties of Hf films produced at the JPL Microdevices Laboratory (MDL).

enclosed in a superconducting box (Fig. 3) to reduce sensitivity to AC magnetic fields. Additional $1 \mathrm{~mm}$ thick high- $\mu$ plates (shown in magenta) are mounted on the faces containing holes for the connectors and flex-circuits; these are intended to direct magnetic field lines around the holes and reduce field ingress.

The daughtercard enclosure was simulated with COMSOL 5.2a. The high-mu material used $\mu_{r}=52000$, which is representative of Amuneal A4K at low temperatures ${ }^{9}$. Superconducting material used $\mu_{r}=10^{-5}$, and magnetic insulation was applied to all superconducting surfaces. The final mesh contained 3.7 million elements. Fig. 4 shows the component of the magnetic field normal to SQUIDs in the SQUID plane for incident magnetic fields along three axes. Note that the shielding can rotate transverse input fields, producing an axial field component near holes in the enclosure. Fig. 5 plots the component of the magnetic field normal to SQUIDs across a single multiplexing column (33 channels) for an axial input field, demonstrating at least a factor of 5000 suppression across the chip. A version of the SQUID enclosure without the high- $\mu$ connector covers shows a factor of 10 worse suppression.

One large high- $\mu$ box, shown in Fig. 3, surrounds the 12 first stage SQUID daughtercards. This box was simulated separately with COMSOL, using the same $\mu_{r}=52000$ as in the previous section. The final mesh contained 316k elements. Fig. 6 shows the total field magnitude in the SQUID plane for incident magnetic fields along three axes. The axial field 

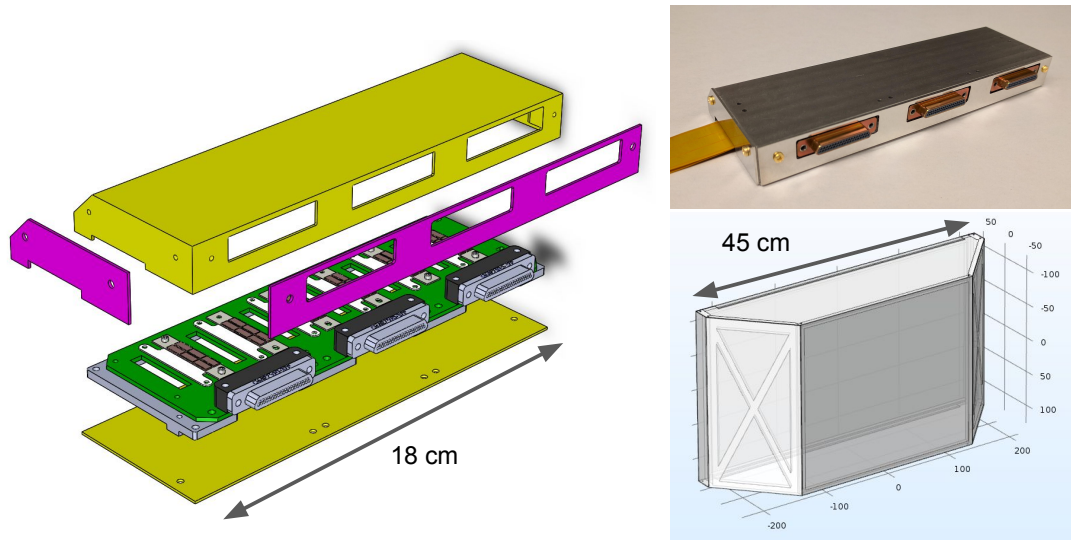

Fig. 3 Left: An exploded render of a TIME SQUID daughtercard housing. Superconducting material (Al) is shown in yellow, and high- $\mu$ metal (A4K) is shown in magenta. Top Right: One of 12 TIME SQUID daughtercards. Bottom Right: The high- $\mu$ box that surrounds the 12 TIME SQUID daughtercards.

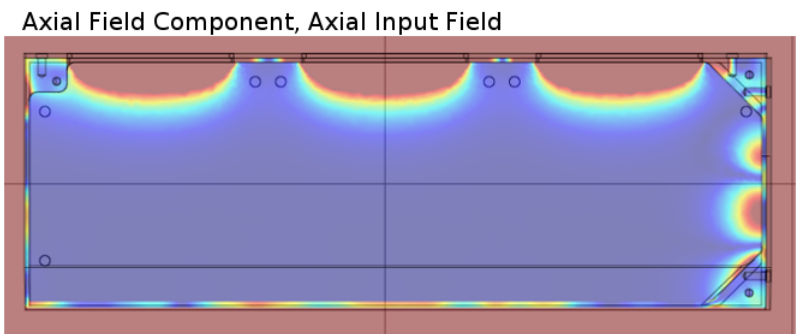

Axial Field Component, Transverse Input Field (Horizontal)

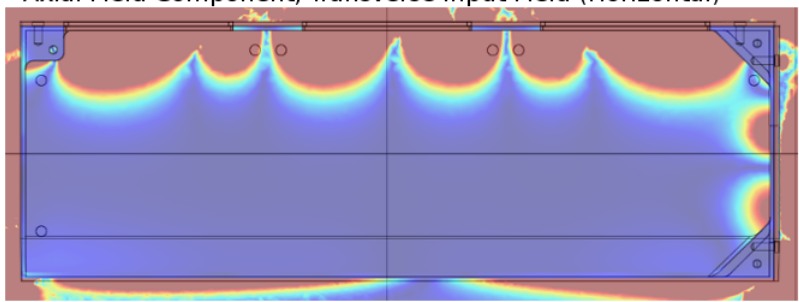

Axial Field Component, Transverse Input Field (Vertical)

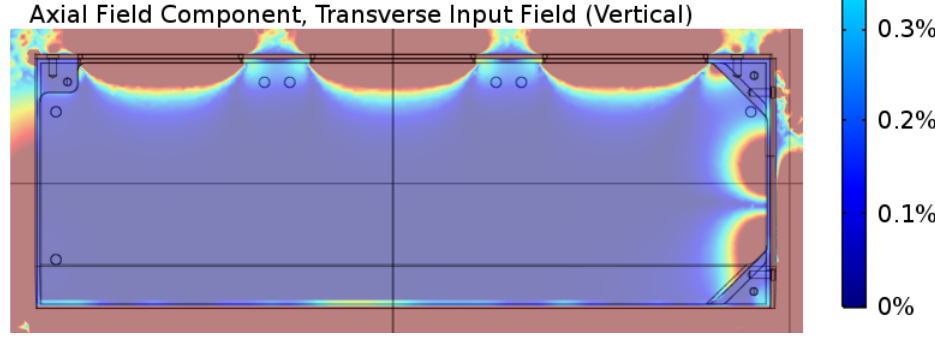

$>1 \%$

$0.9 \%$

$0.8 \%$

$0.7 \%$

$0.6 \%$

$0.5 \%$

$0.4 \%$

$.3 \%$

$0.2 \%$

$0.1 \%$

Fig. 4 COMSOL simulations of the magnetic field suppression of the first stage SQUID daughtercard enclosure (superconducting box with high- $\mu$ connector plates). The axial component of the magnetic field in the plane of the SQUIDs is shown. The three connector holes are located at the top in this view, and the entrance for the superconducting flex-circuit is on the right. 


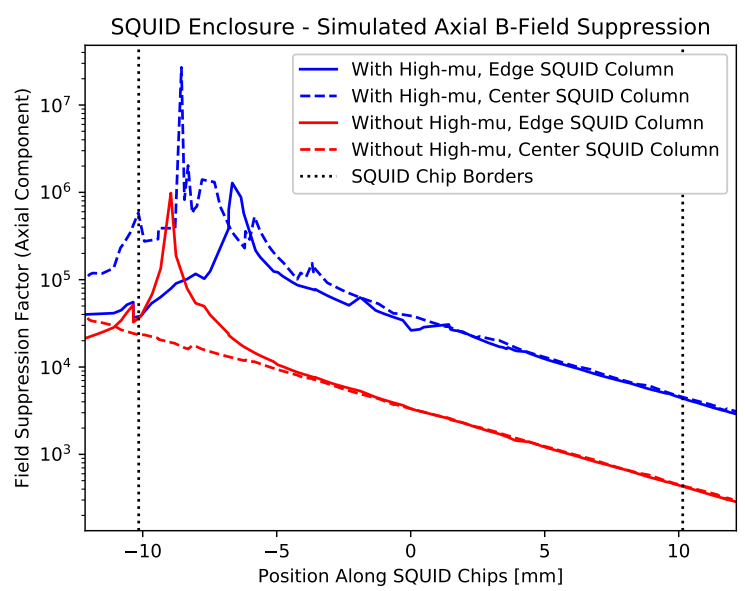

Fig. 5 COMSOL simulations of the magnetic field suppression of the first stage SQUID daughtercard enclosure along the SQUID chips. Addition of high- $\mu$ connector plates (shown in magenta in Fig. 3) increases field suppression by a factor of 10 .

suppression factor is predicted to be $>100$, and the total field suppression factor is predicted to be $>70$ near the SQUIDS.

\subsection{Cryostat Overshield}

A $150 \mathrm{~cm}$ tall high- $\mu$ open-ended cylindrical shield with $1.57 \mathrm{~mm}$ thick walls and a radius of $69 \mathrm{~cm}$ sits within the TIME vacuum shield at 300K. The first stage SQUID modules sit near the center of this shield (67 $\mathrm{cm}$ from the top, $13 \mathrm{~cm}$ away from the shield axis) and are shielded further by the high- $\mu$ and superconducting enclosures described in the previous section. The second stage SQUIDs (at the $4 \mathrm{~K}$ stage) are located closer to the end of the shield ( $26 \mathrm{~cm}$ from the top and $10 \mathrm{~cm}$ from the shield axis) and are enclosed in small $\mathrm{Nb}$ boxes that are wrapped in high- $\mu$ material.

The cylindrical shield was fabricated using Amuneal Amumetal and supression of Earth's magnetic field was measured with a Mag-03MC1000 three-axis magnetic field sensor. Results are plotted in Fig. 7 and are compared to COMSOL simulations using $\mu_{r}=60000$ (the value Amuneal advertises ${ }^{11}$ at $300 \mathrm{~K}$ in $0.5 \mu \mathrm{T}$ magnetic fields). The SQUID normal axes (for both stages) are orthogonal to the shield axis, so fields transverse to the cylindrical shield are the most relevant; the suppression factor near the first stage SQUIDS is approximately 100.

\section{Conclusions}

Hafnium shows promise as a low- $T_{c}$ alternative to Ti for TIME TESs. Fabrication and testing of TIME detectors with Hf TESs is ongoing.

Magnetic shielding for the TIME first-stage SQUIDs has been presented and simulated, with a predicted total axial field suppression factor of at least $5 \times 10^{7}$. For comparison, the achieved magnetic field suppression factor for BICEP2 is quoted ${ }^{10}$ at $\sim 10^{6}$. 


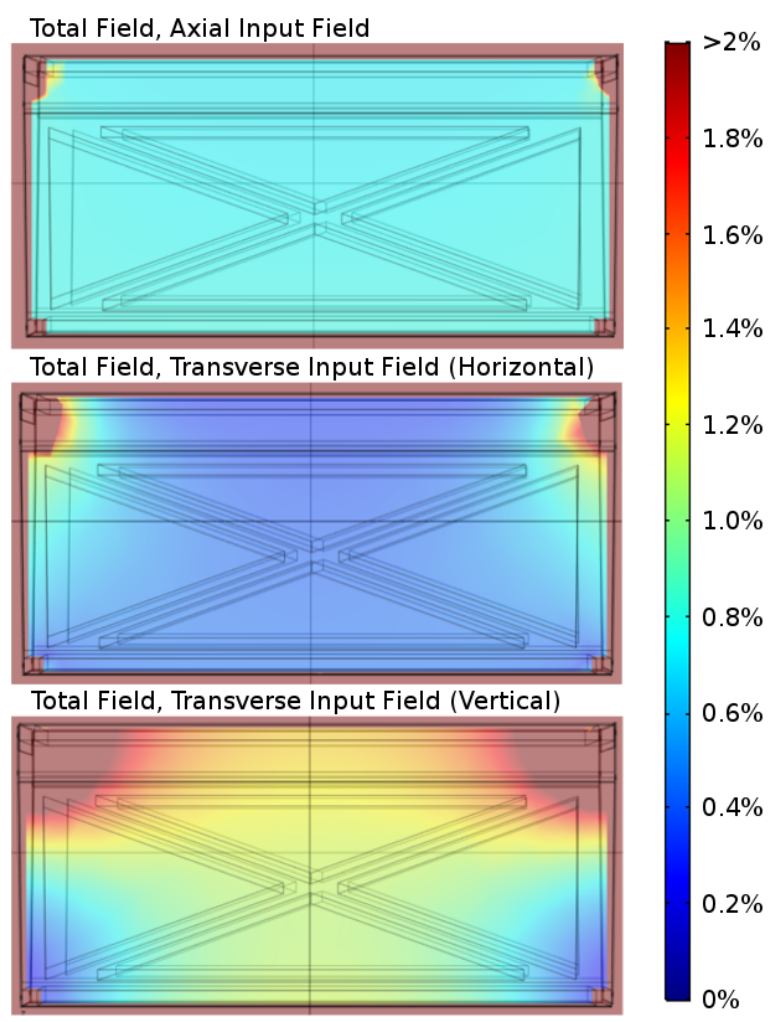

Fig. 6 COMSOL simulations of the magnetic field suppression of the first stage SQUID module overshield (high- $\mu$ box). The total magnitude of the magnetic field in the plane of the SQUIDs of the center daughtercard is shown.

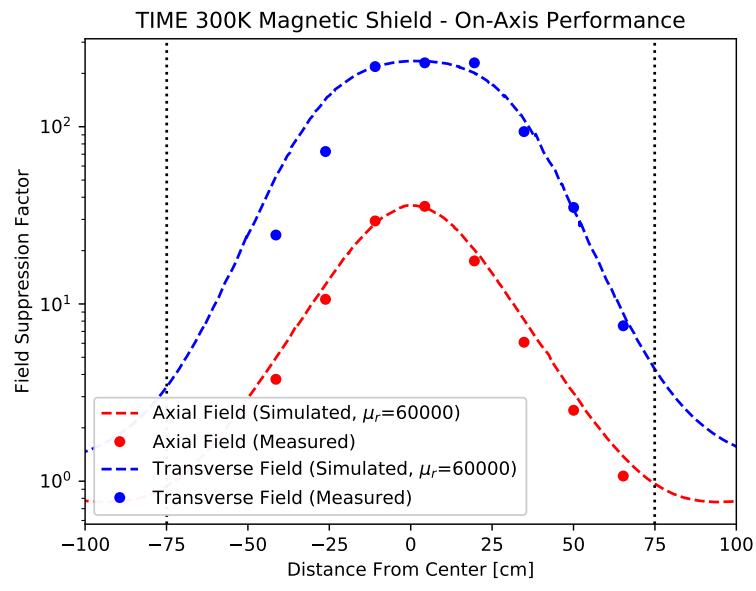

Fig. 7 Measurements of the TIME high- $\mu$ overshield compared to COMSOL simulations with $\mu_{r}=60000$. 
Acknowledgements $\mathrm{JH}$ is supported by the National Science Foundation Graduate Research Fellowship under Grant No. DGE-1144469. AC is supported by the National Science Foundation Astronomy and Astrophysics Post- doctoral Fellowship under Grant No. 1602677.

\section{References}

1. Hunacek, J., Bock, J., Bradford, C. M., et al. 2016, Proc. SPIE, 9914, 99140L

2. Crites, A. T., Bock, J. J., Bradford, C. M., Chang, T. C., Cooray, A. R., Duband, L., Gong, Y., Hailey-Dunsheath, S., Hunacek, J., Koch, P. M., Li, C. T., O’Brient, R. C., Prouve, T., Shirokoff, E., Silva, M. B., Staniszewski, Z., Uzgil, B., and Zemcov, M., "The TIME-Pilot intensity mapping experiment," in [Millimeter, Submillimeter, and Far-Infrared Detectors and Instrumentation for Astronomy VII], Proc. SPIE 9153, 91531W (Aug. 2014).

3. Hunacek, J., Bock, J., Bradford, C. M., Bumble, B., Chang, T.-C., Cheng, Y.-T., Cooray, A., Crites, A., Hailey-Dunsheath, S., Gong, Y., Kenyon, M., Koch, P., Li, C.-T., O’Brient, R., Shirokoff, E., Shiu, C., Staniszewski, Z., Uzgil, B., and Zemcov, M., "Design and fabrication of tes detector modules for the time-pilot [cii] intensity mapping experiment," Journal of Low Temperature Physics 184(3), 733-738 (2016).

4. Li, C.-T., Wei, T., Cheng, J.-C., Shiu, C., Crites, A. T., and Bradford, C. M., "Development of a millimeter wave grating spectrometer for TIME Pilot," The 27th International Symposium on Space Terahertz Technology (2016).

5. Gong, Y., Cooray, A., Silva, M., Santos, M. G., Bock, J., Bradford, C. M., and Zemcov, M., "Intensity Mapping of the [C II] Fine Structure Line during the Epoch of Reionization," ApJ 745, 49 (Jan. 2012).

6. Silva, M., Santos, M. G., Cooray, A., and Gong, Y., "Prospects for Detecting C II Emission during the Epoch of Reionization," ApJ 806, 209 (June 2015).

7. Bradford, C. M., Naylor, B. J., Zmuidzinas, J., Bock, J. J., Gromke, J., Nguyen, H., Dragovan, M., Yun, M., Earle, L., Glenn, J., Matsuhara, H., Ade, P. A. R., and Duband, L., "WaFIRS: a waveguide far-IR spectrometer: enabling spectroscopy of high-z galaxies in the far-IR and submillimeter," in [IR Space Telescopes and Instruments], Mather, J. C., ed., Proc. SPIE 4850, 1137-1148 (Mar. 2003).

8. Bradford, C. M., Aguirre, J. E., Aikin, R., Bock, J. J., Earle, L., Glenn, J., Inami, H., Maloney, P. R., Matsuhara, H., Naylor, B. J., Nguyen, H. T., and Zmuidzinas, J., "The Warm Molecular Gas around the Cloverleaf Quasar," ApJ 705, 112-122 (Nov. 2009).

9. Sah, S., Myneni, G., \& Atulasimha, J. 2015, arXiv:1501.07312

10. BICEP2 Collaboration, Ade, P. A. R., Aikin, R. W., et al. 2014, ApJ, 792, 62

11. http://www.amuneal.com/magnetic-shielding/magnetic-shielding-materials (Retrieved Oct. 10 2017) 